\title{
Distinct Distances in Graph Drawings
}

\author{
Paz Carmi* Vida Dujmović* ${ }^{*}$ Pat Morin* David R. Wood ${ }^{\dagger}$
}

Submitted: Apr 24, 2008; Accepted: Aug 13, 2008; Published: Aug 25, 2008

Mathematics Subject Classification: 05C62 (graph representations)

\begin{abstract}
The distance-number of a graph $G$ is the minimum number of distinct edgelengths over all straight-line drawings of $G$ in the plane. This definition generalises many well-known concepts in combinatorial geometry. We consider the distancenumber of trees, graphs with no $K_{4}^{-}$-minor, complete bipartite graphs, complete graphs, and cartesian products. Our main results concern the distance-number of graphs with bounded degree. We prove that $n$-vertex graphs with bounded maximum degree and bounded treewidth have distance-number in $\mathcal{O}(\log n)$. To conclude such a logarithmic upper bound, both the degree and the treewidth need to be bounded. In particular, we construct graphs with treewidth 2 and polynomial distance-number. Similarly, we prove that there exist graphs with maximum degree 5 and arbitrarily large distance-number. Moreover, as $\Delta$ increases the existential lower bound on the distance-number of $\Delta$-regular graphs tends to $\Omega\left(n^{0.864138}\right)$.
\end{abstract}

\section{Introduction}

This paper initiates the study of the minimum number of distinct edge-lengths in a drawing of a given graph ${ }^{1}$. A degenerate drawing of a graph $G$ is a function that maps the

\footnotetext{
${ }^{*}$ School of Computer Science, Carleton University, Ottawa, Canada (\{paz, vida, morin\}@scs. carleton. ca). Research supported by NSERC.

${ }^{\dagger}$ Department of Mathematics and Statistics, The University of Melbourne, Melbourne, Australia (D.Wood@ms.unimelb.edu.au). Supported by a QEII Research Fellowship. Research initiated at the Universitat Politècnica de Catalunya (Barcelona, Spain), where supported by the Marie Curie Fellowship MEIF-CT-2006-023865, and by the projects MEC MTM2006-01267 and DURSI 2005SGR00692.

${ }^{1}$ We consider graphs that are simple, finite, and undirected. The vertex set of a graph $G$ is denoted by $V(G)$, and its edge set by $E(G)$. A graph with $n$ vertices, $m$ edges and maximum degree at most $\Delta$ is an $n$-vertex, $m$-edge, degree- $\Delta$ graph. A graph in which every vertex has degree $\Delta$ is $\Delta$-regular. For $S \subseteq V(G)$, let $G[S]$ be the subgraph of $G$ induced by $S$, and let $G-S:=G[V(G) \backslash S]$. For each vertex $v \in V(G)$, let $G-v:=G-\{v\}$. Standard notation is used for graphs: complete graphs $K_{n}$, complete bipartite graphs $K_{m, n}$, paths $P_{n}$, and cycles $C_{n}$. A graph $H$ is a minor of a graph $G$ if $H$ can be obtained from a subgraph of $G$ by contracting edges. Throughout the paper, $c$ is a positive constant. Of course, different occurrences of $c$ might denote different constants.
} 
vertices of $G$ to distinct points in the plane, and maps each edge $v w$ of $G$ to the open straight-line segment joining the two points representing $v$ and $w$. A drawing of $G$ is a degenerate drawing of $G$ in which the image of every edge of $G$ is disjoint from the image of every vertex of $G$. That is, no vertex intersects the interior of an edge. In what follows, we often make no distinction between a vertex or edge in a graph and its image in a drawing.

The distance-number of a graph $G$, denoted by $\operatorname{dn}(G)$, is the minimum number of distinct edge-lengths in a drawing of $G$. The degenerate distance-number of $G$, denoted by $\operatorname{ddn}(G)$, is the minimum number of distinct edge-lengths in a degenerate drawing of $G$. Clearly, $\operatorname{ddn}(G) \leq \operatorname{dn}(G)$ for every graph $G$. Furthermore, if $H$ is a subgraph of $G$ then $\operatorname{ddn}(H) \leq \operatorname{ddn}(G)$ and $\operatorname{dn}(H) \leq \operatorname{dn}(G)$.

\subsection{Background and Motivation}

The degenerate distance-number and distance-number of a graph generalise various concepts in combinatorial geometry, which motivates their study.

A famous problem raised by Erdős [15] asks for the minimum number of distinct distances determined by $n$ points in the plane ${ }^{2}$. This problem is equivalent to determining the degenerate distance-number of the complete graph $K_{n}$. We have the following bounds on $\operatorname{ddn}\left(K_{n}\right)$, where the lower bound is due to Katz and Tardos [25] (building on recent advances by Solymosi and Tóth [47], Solymosi et al. [46], and Tardos [50]), and the upper bound is due to Erdős [15].

Lemma 1 ([15, 25]). The degenerate distance-number of $K_{n}$ satisfies

$$
\Omega\left(n^{0.864137}\right) \leq \operatorname{ddn}\left(K_{n}\right) \leq \frac{c n}{\sqrt{\log n}}
$$

Observe that no three points are collinear in a (non-degenerate) drawing of $K_{n}$. Thus $\mathrm{dn}\left(K_{n}\right)$ equals the minimum number of distinct distances determined by $n$ points in the plane with no three points collinear. This problem was considered by Szemerédi (see Theorem 13.7 in [37]), who proved that every such point set contains a point from which there are at least $\left\lceil\frac{n-1}{3}\right\rceil$ distinct distances to the other points. Thus we have the next result, where the upper bound follows from the drawing of $K_{n}$ whose vertices are the points of a regular $n$-gon, as illustrated in Figure 1(a).

Lemma 2 (Szemerédi). The distance-number of $K_{n}$ satisfies

$$
\left\lceil\frac{n-1}{3}\right\rceil \leq \mathrm{dn}\left(K_{n}\right) \leq\left\lfloor\frac{n}{2}\right\rfloor
$$

Note that Lemmas 1 and 2 show that for every sufficiently large complete graph, the degenerate distance-number is strictly less than the distance-number. Indeed, $\operatorname{ddn}\left(K_{n}\right) \in$ $o\left(\operatorname{dn}\left(K_{n}\right)\right)$.

\footnotetext{
${ }^{2}$ For a detailed exposition on distinct distances in point sets refer to Chapters 10-13 of the monograph by Pach and Agarwal [37].
} 
(a)

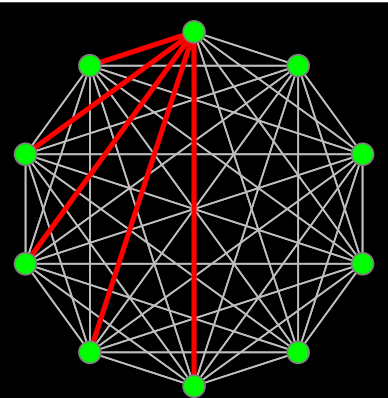

(b)

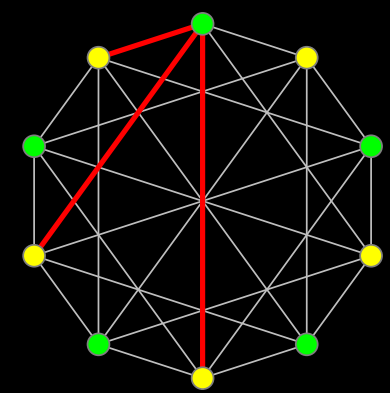

Figure 1: (a) A drawing of $K_{10}$ with five edge-lengths, and (b) a drawing of $K_{5,5}$ with three edge-lengths.

Degenerate distance-number generalises another concept in combinatorial geometry. The unit-distance graph of a set $S$ of points in the plane has vertex set $S$, where two vertices are adjacent if and only if they are at unit-distance; see [23, 35, 36, 39, 42, 45] for example. The famous Hadwiger-Nelson problem asks for the maximum chromatic number of a unit-distance graph. Every unit-distance graph $G$ has $\operatorname{ddn}(G)=1$. But the converse is not true, since a degenerate drawing allows non-adjacent vertices to be at unit-distance. Figure 2 gives an example of a graph $G$ with $\operatorname{dn}(G)=\operatorname{ddn}(G)=1$ that is not a unit-distance graph. In general, $\operatorname{ddn}(G)=1$ if and only if $G$ is isomorphic to a subgraph of a unit-distance graph.

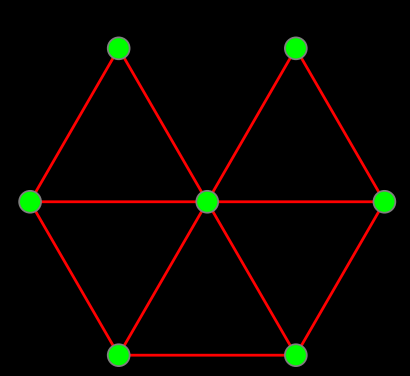

Figure 2: A graph with distance-number 1 that is not a unit-distance graph. In every mapping of the vertices to distinct points in the plane with unit-length edges, $v$ and $w$ are at unit-distance.

The maximum number of edges in a unit-distance graph is an old open problem. The best construction, due to Erdös [15], gives an $n$-vertex unit-distance graph with $n^{1+c / \log \log n}$ edges. The best upper bound on the number of edges is $c n^{4 / 3}$, due to Spencer et al. [48]. (Székely [49] found a simple proof for this upper bound based on the crossing lemma.)

More generally, many recent results in the combinatorial geometry literature provide upper bounds on the number of times the $d$ most frequent inter-point distances can occur 
between a set of $n$ points. Such results are equivalent to upper bounds on the number of edges in an $n$-vertex graph with degenerate distance number $d$. This suggests the following extremal function. Let ex $(n, d)$ be the maximum number of edges in an $n$-vertex graph $G$ with $\operatorname{ddn}(G) \leq d$.

Since every graph $G$ is the union of $\operatorname{ddn}(G)$ subgraphs of unit-distance graphs, the above result by Spencer et al. [48] implies:

Lemma 3 (Spencer et al. [48]).

$$
\operatorname{ex}(n, d) \leq c d n^{4 / 3}
$$

Equivalently, the distance-numbers of every n-vertex m-edge graph $G$ satisfy

$$
\operatorname{dn}(G) \geq \operatorname{ddn}(G) \geq \mathrm{cmn}^{-4 / 3} .
$$

Results by Katz and Tardos [25] (building on recent advances by Solymosi and Tóth [47], Solymosi et al. [46], and Tardos [50]) imply:

Lemma 4 (Katz and Tardos [25]).

$$
\operatorname{ex}(n, d) \in \mathcal{O}\left(n^{1.457341} d^{0.627977}\right) .
$$

Equivalently, the distance-numbers of every $n$-vertex $m$-edge graph $G$ satisfy

$$
\operatorname{dn}(G) \geq \operatorname{ddn}(G) \in \Omega\left(m^{1.592412} n^{-2.320687}\right) .
$$

Note that Lemma 4 improves upon Lemma 3 whenever $\operatorname{ddn}(G)>n^{1 / 3}$. Also note that Lemma 4 implies the lower bound in Lemma 2.

\subsection{Our Results}

The above results give properties of various graphs defined with respect to the inter-point distances of a set of points in the plane. This paper, which is more about graph drawing than combinatorial geometry, reverses this approach, and asks for a drawing of a given graph with few inter-point distances.

Our first results provide some general families of graphs, namely trees and graphs with no $K_{4}^{-}$-minor, that are unit-distance graphs (Section 2). Here $K_{4}^{-}$is the graph obtained from $K_{4}$ by deleting one edge. Then we give bounds on the distance-numbers of complete bipartite graphs (Section 3).

Our main results concern graphs of bounded degree (Section 4). We prove that for all $\Delta \geq 5$ there are degree- $\Delta$ graphs with unbounded distance-number. Moreover, for $\Delta \geq 7$ we prove a polynomial lower bound on the distance-number (of some degree- $\Delta$ graph) that tends to $\Omega\left(n^{0.864138}\right)$ for large $\Delta$. On the other hand, we prove that graphs with bounded degree and bounded treewidth have distance-number in $\mathcal{O}(\log n)$. Note that bounded treewidth alone does not imply a logarithmic bound on distance-number since $K_{2, n}$ has treewidth 2 and degenerate distance-number $\Theta(\sqrt{n})$ (see Section 3 ).

Then we establish an upper bound on the distance-number in terms of the bandwidth (Section 5). Then we consider the distance-number of the cartesian product of graphs (Section 6). We conclude in Section 7 with a discussion of open problems related to distance-number. 


\subsection{Higher-Dimensional Relatives}

Graph invariants related to distances in higher dimensions have also been studied. Erdős, Harary, and Tutte [16] defined the dimension of a graph $G$, denoted by $\operatorname{dim}(G)$, to be the minimum integer $d$ such that $G$ has a degenerate drawing in $\Re^{d}$ with straight-line edges of unit-length. They proved that $\operatorname{dim}\left(K_{n}\right)=n-1$, the dimension of the $n$-cube is 2 for $n \geq 2$, the dimension of the Peterson graph is 2 , and $\operatorname{dim}(G) \leq 2 \cdot \chi(G)$ for every graph $G$. (Here $\chi(G)$ is the chromatic number of $G$.) The dimension of complete 3-partite graphs and wheels were determined by Buckley and Harary [10].

The unit-distance graph of a set $S \subseteq \Re^{d}$ has vertex set $S$, where two vertices are adjacent if and only if they are at unit-distance. Thus $\operatorname{dim}(G) \leq d$ if and only if $G$ is isomorphic to a subgraph of a unit-distance graph in $\Re^{d}$. Maehara [32] proved for all $d$ there is a finite bipartite graph (which thus has dimension at most 4) that is not a unitdistance graph in $\Re^{d}$. This highlights the distinction between dimension and unit-distance graphs. Maehara [32] also proved that every finite graph with maximum degree $\Delta$ is a unit-distance graph in $\Re^{\Delta\left(\Delta^{2}-1\right) / 2}$, which was improved to $\Re^{2 \Delta}$ by Maehara and Rödl [33]. These results are in contrast to our result that graphs of bounded degree have arbitrarily large distance-number.

A graph is $d$-realizable if, for every mapping of its vertices to (not-necessarily distinct) points in $\Re^{p}$ with $p \geq d$, there exists such a mapping in $\Re^{d}$ that preserves edge-lengths. For example, $K_{3}$ is 2-realizable but not 1-realizable. Belk and Connelly [6] and Belk [5] proved that a graph is 2-realizable if and only if it has treewidth at most 2. They also characterized the 3 -realizable graphs as those with no $K_{5}$-minor and no $K_{2,2,2}$-minor.

\section{Some Unit-Distance Graphs}

This section shows that certain families of graphs are unit-distance graphs. The proofs are based on the fact that two distinct circles intersect in at most two points. We start with a general lemma. A graph $G$ is obtained by pasting subgraphs $G_{1}$ and $G_{2}$ on a cut-vertex $v$ of $G$ if $G=G_{1} \cup G_{2}$ and $V\left(G_{1}\right) \cap V\left(G_{2}\right)=\{v\}$.

Lemma 5. Let $G$ be the graph obtained by pasting subgraphs $G_{1}$ and $G_{2}$ on a vertex $v$. Then:

(a) if $\operatorname{ddn}\left(G_{1}\right)=\operatorname{ddn}\left(G_{2}\right)=1$ then $\operatorname{ddn}(G)=1$, and

(b) if $\operatorname{dn}\left(G_{1}\right)=\operatorname{dn}\left(G_{2}\right)=1$ then $\operatorname{dn}(G)=1$.

Proof. We prove part (b). Part (a) is easier. Let $D_{i}$ be a drawing of $G_{i}$ with unit-length edges. Translate $D_{2}$ so that $v$ appears in the same position in $D_{1}$ and $D_{2}$. A rotation of $D_{2}$ about $v$ is bad if its union with $D_{1}$ is not a drawing of $G$. That is, some vertex in $D_{2}$ coincides with the closure of some edge of $D_{1}$, or vice versa. Since $G$ is finite, there are only finitely many bad rotations. Since there are infinitely many rotations, there exists a rotation that is not bad. That is, there exists a drawing of $G$ with unit-length edges.

We have a similar result for unit-distance graphs. 


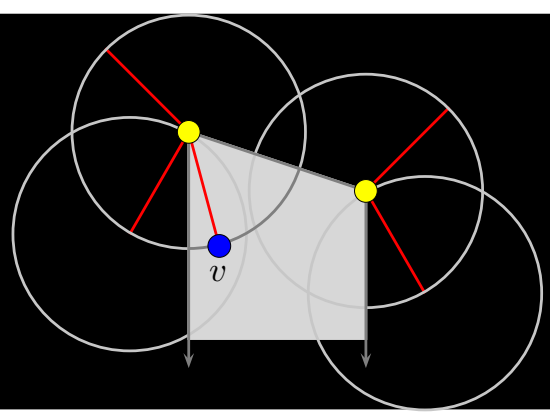

Figure 3: Illustration for the proof of Lemma 7

Lemma 6. Let $G_{1}$ and $G_{2}$ be unit-distance graphs. Let $G$ be the (abstract) graph obtained by pasting $G_{1}$ and $G_{2}$ on a vertex $v$. Then $G$ is isomorphic to a unit-distance graph.

Proof. The proof is similar to the proof of Lemma 5, except that we must ensure that the distance between vertices in $G_{1}-v$ and vertices in $G_{2}-v$ (which are not adjacent) is not 1. Again this will happen for only finitely many rotations. Thus there exists a rotation that works.

Since every tree can be obtained by pasting a smaller tree with $K_{2}$, Lemma 6 implies that every tree is a unit-distance graph. The following is a stronger result.

Lemma 7. Every tree $T$ has a crossing-free ${ }^{3}$ drawing in the plane such that two vertices are adjacent if and only if they are unit-distance apart.

Proof. For a point $v=(\mathrm{x}(v), \mathrm{y}(v))$ in the plane, let $v \downarrow$ be the ray from $v$ to $(\mathrm{x}(v),-\infty)$. We proceed by induction on $n$ with the following hypothesis: Every tree $T$ with $n$ vertices has the desired drawing, such that the vertices have distinct $\mathrm{x}$-coordinates, and for each vertex $u$, the ray $u \downarrow$ does not intersect $T$. The statement is trivially true for $n \leq 2$. For $n>2$, let $v$ be a leaf of $T$ with parent $p$. By induction, $T-v$ has the desired drawing. Let $w$ be a vertex of $T-v$, such that no vertex has its $\mathrm{x}$-coordinate between $\times(p)$ and $\times(w)$. Thus the drawing of $T-v$ does not intersect the open region $R$ of the plane bounded by the two rays $p \downarrow$ and $w \downarrow$, and the segment $p w$. Let $A$ be the intersection of $R$ with the unit-circle centred at $p$. Thus $A$ is a circular arc. Place $v$ on $A$, so that the distance from $v$ to every vertex except $p$ is not 1 . This is possible since $A$ is infinite, and there are only finitely many excluded positions on $A$ (since $A$ intersects a unit-circle centred at a vertex except $p$ in at most two points). Since there are no elements of $T-v$ in $R$, there are no crossings in the resulting drawing and the induction invariants are maintained for all vertices of $T$.

Recall that $K_{4}^{-}$is the graph obtained from $K_{4}$ by deleting one edge.

Lemma 8. Every 2-connected graph $G$ with no $K_{4}^{-}$-minor is a cycle.

\footnotetext{
${ }^{3} \mathrm{~A}$ drawing is crossing-free if no pair of edges intersect.
} 
Proof. Suppose on the contrary that $G$ has a vertex $v$ of degree at least 3 . Let $x, y, z$ be the neighbours of $v$. There is an $x y$-path $P$ avoiding $v$ (since $G$ is 2-connected) and avoiding $z$ (since $G$ is $K_{4}^{-}$-minor free). Similarly, there is an $x z$-path $Q$ avoiding $v$. If $x$ is the only vertex in both $P$ and $Q$, then the cycle $(x, P, y, v, z, Q)$ plus the edge $x v$ is a subdivision of $K_{4}^{-}$. Now assume that $P$ and $Q$ intersect at some other vertex. Let $t$ be the first vertex on $P$ starting at $x$ that is also in $Q$. Then the cycle $(x, Q, z, v)$ plus the sub-path of $P$ between $x$ and $t$ is a subdivision of $K_{4}^{-}$. This contradiction proves that $G$ has no vertex of degree at least 3 . Since $G$ is 2 -connected, $G$ is a cycle, as desired.

Theorem 1. Every $K_{4}^{-}$-minor-free graph $G$ has a drawing such that vertices are adjacent if and only if they are unit-distance apart. In particular, $G$ is isomorphic to a unit-distance graph and $\operatorname{ddn}(G)=\operatorname{dn}(G)=1$.

Proof. By Lemma 6, we can assume that $G$ is 2-connected. Thus $G$ is a cycle by Lemma 8 . The result follows since $C_{n}$ is a unit-distance graph (draw a regular $n$-gon).

\section{Complete Bipartite Graphs}

This section considers the distance-numbers of complete bipartite graphs $K_{m, n}$. Since $K_{1, n}$ is a tree, $\operatorname{ddn}\left(K_{1, n}\right)=\operatorname{dn}\left(K_{1, n}\right)=1$ by Lemma 7 . The next case, $K_{2, n}$, is also easily handled.

Lemma 9. The distance-numbers of $K_{2, n}$ satisfy

$$
\operatorname{ddn}\left(K_{2, n}\right)=\operatorname{dn}\left(K_{2, n}\right)=\left\lceil\sqrt{\frac{n}{2}}\right\rceil .
$$

Proof. Let $G=K_{2, n}$ with colour classes $A=\{v, w\}$ and $B$, where $|B|=n$. We first prove the lower bound, $\operatorname{ddn}\left(K_{2, n}\right) \geq\left\lceil\sqrt{\frac{n}{2}}\right\rceil$. Consider a degenerate drawing of $G$ with $\operatorname{ddn}(G)$ edge-lengths. The vertices in $B$ lie on the intersection of $\operatorname{ddn}(G)$ concentric circles centered at $v$ and $\operatorname{ddn}(G)$ concentric circles centered at $w$. Since two distinct circles intersect in at most two points, $n \leq 2 \mathrm{ddn}(G)^{2}$. Thus $\operatorname{ddn}\left(K_{2, n}\right) \geq\left\lceil\sqrt{\frac{n}{2}}\right\rceil$.

For the upper bound, position $v$ at $(-1,0)$ and $w$ at $(1,0)$. As illustrated in Figure 4, draw $\left\lceil\sqrt{\frac{n}{2}}\right\rceil$ circles centered at each of $v$ and $w$ with radii ranging strictly between 1 and 2 , such that the intersections of the circles together with $v$ and $w$ define a set of points with no three points collinear. (This can be achieved by choosing the radii iteratively, since for each circle $C$, there are finitely many forbidden values for the radius of $C$.) Each pair of non-concentric circles intersect in two points. Thus the number of intersection points is at least $n$. Placing the vertices of $B$ at these intersection points results in a drawing of $K_{2, n}$ with $\left\lceil\sqrt{\frac{n}{2}}\right\rceil$ edge-lengths.

Now we determine $\operatorname{ddn}\left(K_{3, n}\right)$ to within a constant factor.

Lemma 10. The degenerate distance-number of $K_{3, n}$ satisfies

$$
\left\lceil\sqrt{\frac{n}{2}}\right\rceil \leq \mathrm{ddn}\left(K_{3, n}\right) \leq 3\left\lceil\sqrt{\frac{n}{2}}\right\rceil-1 .
$$




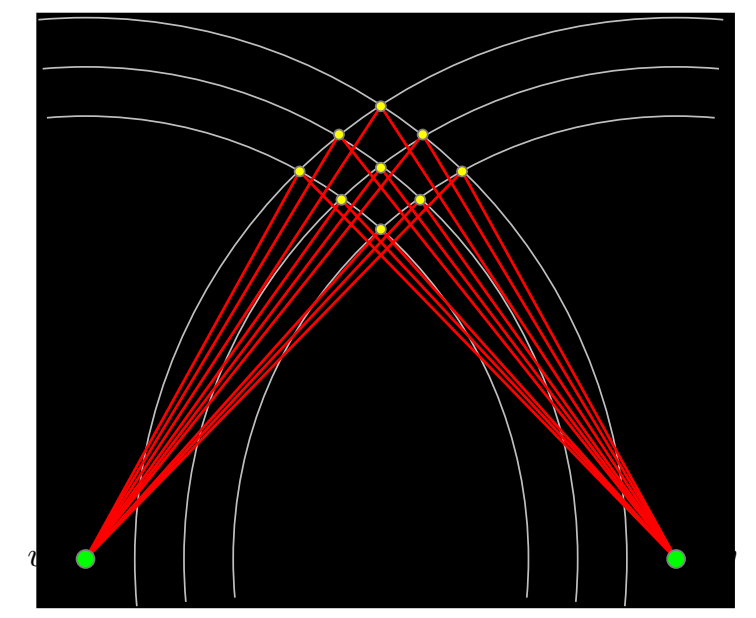

Figure 4: Illustration for the proof of Lemma 9.

Proof. The lower bound follows from Lemma 9 since $K_{2, n}$ is a subgraph of $K_{3, n}$.

Now we prove the upper bound. Let $A$ and $B$ be the colour classes of $K_{3, n}$, where $|A|=3$ and $|B|=n$. Place the vertices in $A$ at $(-1,0),(0,0)$, and $(1,0)$. Let $d:=\left\lceil\sqrt{\frac{n}{2}}\right\rceil$. For $i \in[d]$, let

$$
r_{i}:=\sqrt{1+\frac{i}{d+1}} .
$$

Note that $1<r_{i}<2$. Let $R_{i}$ be the circle centred at $(-1,0)$ with radius $r_{i}$. For $j \in[d]$, let $S_{j}$ be the circle centred at $(1,0)$ with radius $r_{j}$. Observe that each pair of circles $R_{i}$ and $S_{j}$ intersect in exactly two points. Place the vertices in $B$ at the intersection points of these circles. This is possible since $2 d^{2} \geq n$.

Let $(x, y)$ and $(x,-y)$ be the two points where $R_{i}$ and $S_{j}$ intersect. Thus $(x+1)^{2}+y^{2}=$ $r_{i}^{2}$ and $(x-1)^{2}+y^{2}=r_{j}^{2}$. It follows that

$$
x^{2}+y^{2}=\frac{i}{d+1}+2 x=\frac{j}{d+1}-2 x
$$

Thus $2\left(x^{2}+y^{2}\right)=\frac{i+j}{d+1}$. That is, the distance from $(x, y)$ to $(0,0)$ equals

$$
\sqrt{\frac{i+j}{2 d+2}}
$$

which is the same distance from $(x,-y)$ to $(0,0)$. Thus the distance from each vertex in $B$ to $(0,0)$ is one of $2 d-1$ values (determined by $i+j)$. The distance from each vertex in $B$ to $(-1,0)$ and to $(1,0)$ is one of $d$ values. Hence the degenerate distance-number of $K_{3, n}$ is at most $3 d-1=3\left\lceil\sqrt{\frac{n}{2}}\right\rceil-1$.

Now consider the distance-number of a general complete bipartite graph. 


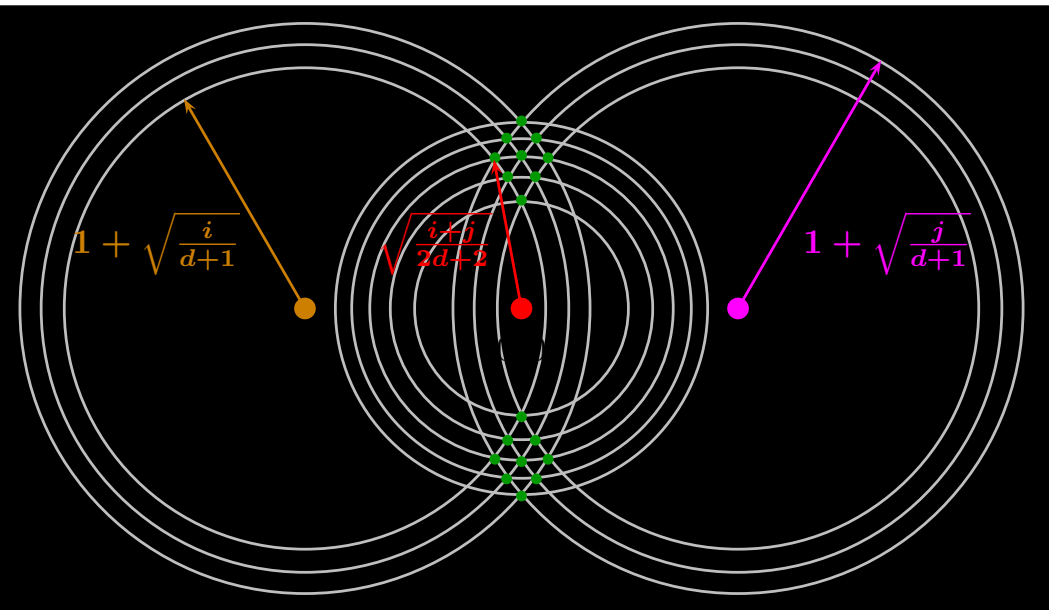

Figure 5: Illustration for the proof of Lemma 10.

Lemma 11. For all $n \geq m$, the distance-numbers of $K_{m, n}$ satisfy

$$
\Omega\left(\frac{m n}{(m+n)^{1.457341}}\right)^{(1 / 0.627977)} \leq \mathrm{ddn}\left(K_{m, n}\right) \leq \operatorname{dn}\left(K_{m, n}\right) \leq\left\lceil\frac{n}{2}\right\rceil
$$

In particular,

$$
\Omega\left(n^{0.864137}\right) \leq \operatorname{ddn}\left(K_{n, n}\right) \leq \operatorname{dn}\left(K_{n, n}\right) \leq\left\lceil\frac{n}{2}\right\rceil .
$$

Proof. The lower bounds follow from Lemma 4. For the upper bound on $\operatorname{dn}\left(K_{n, n}\right)$, position the vertices on a regular $2 n$-gon $\left(v_{1}, v_{2}, \ldots, v_{2 n}\right)$ alternating between the colour classes, as illustrated in Figure 1(b). In the resulting drawing of $K_{n, n}$, the number of edge-lengths is $\left|\left\{(i+j) \bmod n: v_{i} v_{j} \in E\left(K_{n, n}\right)\right\}\right|$. Since $v_{i} v_{j}$ is an edge if and only if $i+j$ is odd, the number of edge-lengths is $\left\lceil\frac{n}{2}\right\rceil$. The upper bound on $\operatorname{dn}\left(K_{n, m}\right)$ follows since $K_{n, m}$ is a subgraph of $K_{n, n}$.

\section{Bounded degree graphs}

Lemma 9 implies that if a graph has two vertices with many common neighbours then its distance-number is necessarily large. Thus it is natural to ask whether graphs of bounded degree have bounded distance-number. This section provides a negative answer to this question.

\subsection{Bounded degree graphs with $\Delta \geq 7$}

This section proves that for all $\Delta \geq 7$ there are $\Delta$-regular graphs with unbounded distancenumber. Moreover, the lower bound on the distance-number is polynomial in the number of vertices. The basic idea of the proof is to show that there are more $\Delta$-regular graphs 
than graphs with bounded distance-number; see $[4,13,14,38]$ for other examples of this paradigm.

It will be convenient to count labelled graphs. Let $\mathcal{G}\langle n, \Delta\rangle$ denote the family of labelled $\Delta$-regular $n$-vertex graphs. Let $\mathcal{G}\langle n, m, d\rangle$ denote the family of labelled $n$-vertex $m$-edge graphs with degenerate distance-number at most $d$. Our results follow by comparing a lower bound on $|\mathcal{G}\langle n, \Delta\rangle|$ with an upper bound on $|\mathcal{G}\langle n, m, d\rangle|$ with $m=\frac{\Delta n}{2}$, which is the number of edges in a $\Delta$-regular $n$-vertex graph.

The lower bound in question is known. In particular, the first asymptotic bounds on the number of labelled $\Delta$-regular $n$-vertex graphs were independently determined by Bender and Canfield [7] and Wormald [52]. McKay [34] further refined these results. We will use the following simple lower bound derived by Barát et al. [4] from the result of McKay [34].

Lemma $12([4,7,34,52])$. For all integers $\Delta \geq 1$ and $n \geq c \Delta$, the number of labelled $\Delta$-regular $n$-vertex graphs satisfies

$$
|\mathcal{G}\langle n, \Delta\rangle| \geq\left(\frac{n}{3 \Delta}\right)^{\Delta n / 2}
$$

The proof of our upper bound on $|\mathcal{G}\langle n, m, d\rangle|$ uses the following special case of the Milnor-Thom theorem by Rónyai et al. [43]. Let $\mathcal{P}=\left(P_{1}, P_{2}, \ldots, P_{t}\right)$ be a sequence of polynomials on $p$ variables over $\Re$. The zero-pattern of $\mathcal{P}$ at $u \in \Re^{p}$ is the set $\{i: 1 \leq$ $\left.i \leq t, P_{i}(u)=0\right\}$.

Lemma $13([43])$. Let $\mathcal{P}=\left(P_{1}, P_{2}, \ldots, P_{t}\right)$ be a sequence of polynomials of degree at most $\delta \geq 1$ on $p \leq t$ variables over $\Re$. Then the number of zero-patterns of $\mathcal{P}$ is at most $\left(\begin{array}{c}\delta t \\ p\end{array}\right)$.

Recall that $\operatorname{ex}(n, d)$ is the maximum number of edges in an $n$-vertex graph $G$ with $\operatorname{ddn}(G) \leq d$. Bounds on this function are given in Lemmas 3 and 4 . Our upper bound on $|\mathcal{G}\langle n, m, d\rangle|$ is expressed in terms of $\operatorname{ex}(n, d)$.

Lemma 14. The number of labelled $n$-vertex $m$-edge graphs with $\mathrm{ddn}(G) \leq d$ satisfies

$$
|\mathcal{G}\langle n, m, d\rangle| \leq\left(\frac{\mathrm{e} n d}{2}\right)^{2 n+d}\left(\begin{array}{c}
\operatorname{ex}(n, d) \\
m
\end{array}\right),
$$

where e is the base of the natural logarithm.

Proof. Let $V(G)=\{1,2, \ldots, n\}$ for every $G \in \mathcal{G}\langle n, m, d\rangle$. For every $G \in \mathcal{G}\langle n, m, d\rangle$, there is a point set

$$
S(G)=\left\{\left(x_{i}(G), y_{i}(G)\right): 1 \leq i \leq n\right\}
$$

and a set of edge-lengths

$$
L(G)=\left\{\ell_{k}(G): 1 \leq k \leq d\right\},
$$

such that $G$ has a degenerate drawing in which each vertex $i$ is represented by the point $\left(x_{i}(G), y_{i}(G)\right)$ and the length of each edge in $E(G)$ is in $L(G)$. Fix one such degenerate drawing of $G$. 
For all $i, j, k$ with $1 \leq i<j \leq n$ and $1 \leq k \leq d$, and for every graph $G \in \mathcal{G}\langle n, m, d\rangle$, define

$$
P_{i, j, k}(G):=\left(x_{j}(G)-x_{i}(G)\right)^{2}+\left(y_{j}(G)-y_{i}(G)\right)^{2}-\ell_{k}(G)^{2} .
$$

Consider $\mathcal{P}:=\left\{P_{i, j, k}: 1 \leq i<j \leq n, 1 \leq k \leq d\right\}$ to be a set of $\left(\begin{array}{l}n \\ 2\end{array}\right) d$ degree-2 polynomials on the set of $2 n+d$ variables $\left\{x_{1}, x_{2}, \ldots, x_{n}, y_{1}, y_{2}, \ldots, y_{n}, \ell_{1}, \ell_{2}, \ldots, \ell_{d}\right\}$. Observe that

$P_{i, j, k}(G)=0$ if and only if the distance between vertices $i$ and $j$ in $\quad(\star)$

the

degenerate drawing of $G$ is $\ell_{k}(G)$.

Recall the well-known fact that $\left(\begin{array}{l}a \\ b\end{array}\right) \leq\left(\frac{\mathrm{e} a}{b}\right)^{b}$. By Lemma 13 with $t=\left(\begin{array}{l}n \\ 2\end{array}\right) d, \delta=2$ and $p=2 n+d$, the number of zero-patterns determined by $\mathcal{P}$ is at most

$$
\left(\begin{array}{c}
2\left(\begin{array}{c}
n \\
2
\end{array}\right) d \\
2 n+d
\end{array}\right) \leq\left(\frac{2 \mathrm{e}\left(\begin{array}{c}
n \\
2
\end{array}\right) d}{2 n+d}\right)^{2 n+d}<\left(\frac{\mathrm{e} n^{2} d}{2 n+d}\right)^{2 n+d}<\left(\frac{\mathrm{e} n^{2} d}{2 n}\right)^{2 n+d}=\left(\frac{\mathrm{e} n d}{2}\right)^{2 n+d}
$$

Fix a zero-pattern $\sigma$ of $\mathcal{P}$. Let $\mathcal{G}_{\sigma}$ be the set of graphs $G$ in $\mathcal{G}\langle n, m, d\rangle$ such that $\sigma$ is the zero-pattern of $\mathcal{P}$ evaluated at $G$. To bound $|\mathcal{G}\langle n, m, d\rangle|$ we now bound $\left|\mathcal{G}_{\sigma}\right|$. Let $H_{\sigma}$ be the graph with vertex set $V\left(H_{\sigma}\right)=\{1, \ldots, n\}$ and edge set $E\left(H_{\sigma}\right)$ where $i j \in E\left(H_{\sigma}\right)$ if and only if $i j \in E(G)$ for some $G \in \mathcal{G}_{\sigma}$. Consider a degenerate drawing of an arbitrary graph $G \in \mathcal{G}_{\sigma}$ on the point set $S(G)$. By $(\star), S(G)$ and $L(G)$ define a degenerate drawing of $H$ with $d$ edge-lengths. Thus $\operatorname{ddn}\left(H_{\sigma}\right) \leq d$ and by assumption, $\left|E\left(H_{\sigma}\right)\right| \leq \operatorname{ex}(n, d)$. Since every graph in $\mathcal{G}_{\sigma}$ is a subgraph of $H_{\sigma},\left|\mathcal{G}_{\sigma}\right| \leq\left(\begin{array}{c}\left|E\left(H_{\sigma}\right)\right| \\ m\end{array}\right)$. Therefore,

$$
|\mathcal{G}\langle n, m, d\rangle| \leq\left(\frac{\mathrm{e} n d}{2}\right)^{2 n+d}\left(\begin{array}{c}
\left|E\left(H_{\sigma}\right)\right| \\
m
\end{array}\right) \leq\left(\frac{\mathrm{e} n d}{2}\right)^{2 n+d}\left(\begin{array}{c}
\mathrm{ex}(n, d) \\
m
\end{array}\right),
$$

as required.

By comparing the lower bound in Lemma 12 and the upper bound in Lemma 14 we obtain the following result.

Lemma 15. Suppose that for some real numbers $\alpha$ and $\beta$ with $\beta>0$ and $1<\alpha<2<$ $\alpha+\beta$,

$$
\operatorname{ex}(n, d) \in \mathcal{O}\left(n^{\alpha} d^{\beta}\right)
$$

Then for every integer $\Delta>\frac{4}{2-\alpha}$, for all $\varepsilon>0$, and for all sufficiently large $n>$ $n(\alpha, \beta, \Delta, \varepsilon)$, there exists a $\Delta$-regular $n$-vertex graph $G$ with degenerate distance-number

$$
\operatorname{ddn}(G)>n^{\frac{2-\alpha}{\beta}-\frac{(2-\alpha+\beta)(4+2 \varepsilon)}{\beta^{2} \Delta+4 \beta}} .
$$

Proof. In this proof, $\alpha, \beta, \Delta$ and $\epsilon$ are fixed numbers satisfying the assumptions of the lemma. Let $d$ be the maximum degenerate distance number of a graph in $\mathcal{G}\langle n, \Delta\rangle$. The result will follow by showing that for all sufficiently large $n>n(\alpha, \beta, \Delta, \varepsilon)$,

$$
d>n^{\frac{2-\alpha}{\beta}-\frac{(2-\alpha+\beta)(4+2 \varepsilon)}{\beta^{2} \Delta+4 \beta}} .
$$


By the definition of $d$, and since every $\Delta$-regular $n$-vertex graph has $\frac{\Delta n}{2}$ edges, every graph in $\mathcal{G}\langle n, \Delta\rangle$ is also in $\mathcal{G}\left\langle n, \frac{\Delta n}{2}, d\right\rangle$. By Lemma 12 with $n \geq c \Delta$, and by Lemma 14 ,

$$
\left(\frac{n}{3 \Delta}\right)^{\Delta n / 2} \leq|\mathcal{G}\langle n, \Delta\rangle| \leq\left|\mathcal{G}\left\langle n, \frac{\Delta n}{2}, d\right\rangle\right| \leq\left(\frac{\mathrm{e} n d}{2}\right)^{2 n+d}\left(\begin{array}{c}
\operatorname{ex}(n, d) \\
\Delta n / 2
\end{array}\right) .
$$

Since $\operatorname{ex}(n, d) \in \mathcal{O}\left(n^{\alpha} d^{\beta}\right)$, and since $d$ is a function of $n$, there is a constant $c$ such that $\operatorname{ex}(n, d) \leq c n^{\alpha} d^{\beta}$ for sufficiently large $n$. Thus (and since $\left.\left(\begin{array}{l}a \\ b\end{array}\right) \leq\left(\frac{\mathrm{e} a}{b}\right)^{b}\right)$,

$$
\left(\frac{n}{3 \Delta}\right)^{\Delta n / 2} \leq\left(\frac{\mathrm{e} n d}{2}\right)^{2 n+d}\left(\begin{array}{c}
c n^{\alpha} d^{\beta} \\
\Delta n / 2
\end{array}\right) \leq\left(\frac{\mathrm{e} n d}{2}\right)^{2 n+d}\left(\frac{2 \mathrm{e} c n^{\alpha} d^{\beta}}{\Delta n}\right)^{\Delta n / 2}
$$

Hence

$$
n^{\Delta n} \leq 3^{\Delta n}\left(\frac{\mathrm{e} n d}{2}\right)^{4 n+2 d}\left(2 \mathrm{e} c n^{\alpha-1} d^{\beta}\right)^{\Delta n} .
$$

By Lemma $2, d \leq \operatorname{ddn}\left(K_{n}\right) \leq \frac{c n}{\sqrt{\log n}}$, implying $2 d \leq \varepsilon n$ for all large $n>n(\varepsilon)$. Thus

$$
n^{\Delta} \leq 3^{\Delta}\left(\frac{\mathrm{e} n d}{2}\right)^{4+\varepsilon}\left(2 \mathrm{ecn}{ }^{\alpha-1} d^{\beta}\right)^{\Delta} .
$$

Hence

$$
n^{(2-\alpha) \Delta-4-\varepsilon} \leq 3^{\Delta}\left(\frac{\mathrm{e}}{2}\right)^{4+\varepsilon}(2 \mathrm{e} c)^{\Delta} d^{\beta \Delta+4+\varepsilon} .
$$

Observe that $3^{\Delta}\left(\frac{\mathrm{e}}{2}\right)^{4+\varepsilon}(2 \mathrm{e} c)^{\Delta} \leq n^{\varepsilon}$ for all large $n>n(\Delta, \varepsilon)$. Thus

$$
n^{(2-\alpha) \Delta-4-2 \varepsilon} \leq d^{\beta \Delta+4+\varepsilon} \text {. }
$$

Hence

$$
d \geq n^{\frac{(2-\alpha) \Delta-4-2 \varepsilon}{\beta \Delta+4+\varepsilon}}=n^{\frac{2-\alpha}{\beta}-\frac{(2-\alpha+\beta)(4+\varepsilon)+\beta \epsilon}{\beta(\beta \Delta+4+\varepsilon)}}>n^{\frac{2-\alpha}{\beta}-\frac{(2-\alpha+\beta)(4+2 \varepsilon)}{\beta^{2} \Delta+4 \beta}}
$$

as required.

We can now state the main results of this section. By Lemma 3, the conditions of Lemma 15 are satisfied with $\alpha=\frac{4}{3}$ and $\beta=1$; thus together they imply:

Theorem 2. For every integer $\Delta \geq 7$, for all $\varepsilon>0$, and for all sufficiently large $n>$ $n(\Delta, \varepsilon)$, there exists a $\Delta$-regular $n$-vertex graph $G$ with degenerate distance-number

$$
\operatorname{ddn}(G)>n^{\frac{2}{3}-\frac{20+10 \varepsilon}{3 \Delta+12}} .
$$

By Lemma 4, the conditions of Lemma 15 are satisfied with $\alpha=1.457341$ and $\beta=$ 0.627977; thus together they imply:

Theorem 3. For every integer $\Delta \geq 8$, for all $\varepsilon>0$, and for all sufficiently large $n>$ $n(\Delta, \varepsilon)$, there exists a $\Delta$-regular $n$-vertex graph $G$ with degenerate distance-number

$$
\operatorname{ddn}(G)>n^{0.864138-\frac{4.682544+2.341272 \varepsilon}{0.394355 \Delta+2.511908}} .
$$

Note that the bound given in Theorem 3 is better than the bound in Theorem 2 for $\Delta \geq 17$. 


\subsection{Bounded degree graphs with $\Delta \geq 5$}

Theorem 2 shows that for $\Delta \geq 7$ and for sufficiently large $n$, there is an $n$-vertex degree- $\Delta$ graph whose degenerate distance-number is at least polynomial in $n$. We now prove that the degenerate distance-number of degree- 5 graphs can also be arbitrarily large. However, the lower bound we obtain in this case is polylogarithmic in $n$. The proof is inspired by an analogous proof about the slope-number of degree- 5 graphs, due to Pach and Pálvölgyi [38].

Theorem 4. For all $d \in \mathbb{N}$, there is a degree-5 graph $G$ with degenerate distance-number $\operatorname{ddn}(G)>d$.

Proof. Consider the following degree-5 graph $G$. For $n \equiv 0(\bmod 6)$, let $F$ be the graph with vertex set $\left\{v_{1}, v_{2}, \ldots, v_{n}\right\}$ and edge set $\left\{v_{i} v_{j}:|i-j| \leq 2\right\}$. Let $S:=\left\{v_{i}: i \equiv 1\right.$ $(\bmod 3)\}$. No pair of vertices in $S$ are adjacent in $F$, and $|S|=\frac{n}{3}$ is even.

Let $\mathcal{M}$ denote the set of all perfect matchings on $S$. For each perfect matching $M_{k} \in \mathcal{M}$, let $G_{k}:=F \cup M_{k}$. As illustrated in Figure 6 , let $G$ be the disjoint union of all the $G_{k}$. Thus the number of connected components of $G$ is $|\mathcal{M}|$, which is at least $\left(\frac{n}{9}\right)^{n / 6}$ by Lemma 12 with $\Delta=1$. Here we consider perfect matchings to be 1-regular graphs. (It is remarkable that even with $\Delta=1$, Lemma 12 gives such an accurate bound, since the actual number of matchings in $S$ is $\sqrt{2}\left(\frac{n}{3 \mathrm{e}}\right)^{n / 6}$ ignoring lower order additive terms ${ }^{4}$.)
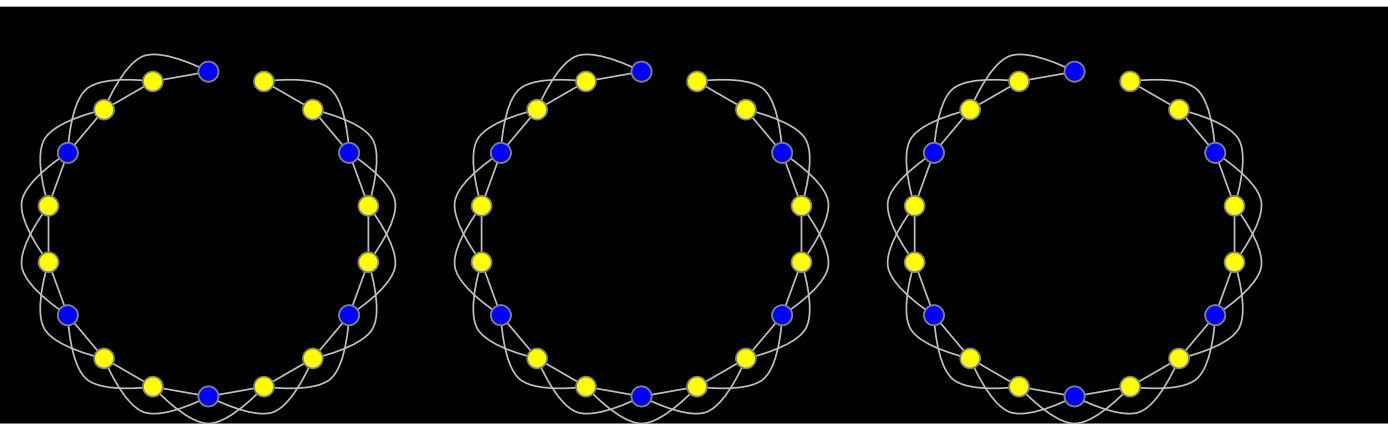

Figure 6: The graph $G$ with $n=18$.

Suppose, for the sake of contradiction, that for some constant $d$, for all $n \in \mathbb{N}$ such that $n \equiv 0(\bmod 6), G$ has a degenerate drawing $D$ with at most $d$ edge-lengths.

Label the edges of $G$ that are in the copies of $F$ by their length in $D$. Let $\ell_{k}(i, j)$ be the label of the edge $v_{i} v_{j}$ in the copy of $F$ in the component $G_{k}$ of $G$. This defines a

\footnotetext{
${ }^{4}$ For even $n$, let $f(n)$ be the number of perfect matchings of $[n]$. Here we determine the asymptotics of $f$. In every such matching, $n$ is matched with some number in $[n-1]$, and the remaining matching is isomorphic to a perfect matching of $[n-2]$. Every matching obtained in this way is distinct. Thus $f(n)=(n-1) \cdot f(n-2)$, where $f(2)=1$. Hence $f(n)=(n-1) ! !=(n-1)(n-3)(n-5) \ldots 1$, where !! is the double factorial function. Now $(2 n-1) ! !=\frac{(2 n) !}{2^{n} n !}$. Thus $f(n)=\frac{n !}{2^{n / 2}(n / 2) !} \approx \sqrt{2}\left(\frac{n}{\mathrm{e}}\right)^{n / 2}$ by Stirling's Approximation.
} 
labelling of the components of $G$. Since $F$ has $2 n-3$ edges and each edge in $F$ receives one of $d$ labels, there are at most $d^{2 n-3}$ distinct labellings of the components of $G$.

Let $D_{k}$ be the degenerate drawing of $G_{k}$ obtained from $D$ by a translation and rotation so that $v_{1}$ is at $(0,0)$ and $v_{2}$ is at $\left(\ell_{k}(1,2), 0\right)$. We say that two components $G_{q}$ and $G_{r}$ of $G$ determine the same set of points if for all $i \in[n]$, the vertex $v_{i}$ in $D_{q}$ is at the same position as the vertex $v_{i}$ in $D_{r}$.

Partition the components of $G$ into the minimum number of parts such that all the components in each part have the same labelling and determine the same set of points.

Observe that two components of $G$ with the same labelling do not necessarily determine the same set of points. However, the number of point sets determined by the components with a given labelling can be bounded as follows. For each component $G_{k}$ of $G, v_{1}$ is at $(0,0)$ and $v_{2}$ is at $\left(\ell_{k}(i, j), 0\right)$ in $D_{k}$. Thus for a fixed labelling, the positions of $v_{1}$ and $v_{2}$ in $D_{k}$ are determined. Now for $i \geq 3$, in each component $G_{k}$, the vertex $v_{i}$ is positioned in $D_{k}$ at the intersection of the circle of radius $\ell_{k}(i-1, i)$ centered at $v_{i-1}$ and the circle of radius $\ell_{k}(i-2, i)$ centered at $v_{i-2}$. Thus there are at most two possible locations for $v_{i}$ (for a fixed labelling). Hence the components with the same labelling determine at most $2^{n-2}$ distinct points sets. Therefore the number of parts in the partition is at most $d^{2 n-3} \cdot 2^{n-2}<\left(2 d^{2}\right)^{n}$.

Finally, we bound the number of components in each part, $R$, of the partition. Let $H_{R}$ be the graph with vertex set $V\left(H_{R}\right)=\left\{v_{1}, \ldots, v_{n}\right\}$ where $v_{i} v_{j} \in E\left(H_{R}\right)$ if and only if $v_{i} v_{j} \in E\left(G_{k}\right)$ for some component $G_{k} \in R$. Since the graphs in $R$ determine the same set of points, the union of the degenerate drawings $D_{k}$, over all $G_{k} \in R$, determines a degenerate drawing of $H_{R}$ with $d$ edge-lengths. Thus $\operatorname{ddn}\left(H_{R}\right) \leq d$ and by Lemma 3, $\left|E\left(H_{R}\right)\right| \leq c d n^{4 / 3}$ for some constant $c>0$. Every component in $R$ is a subgraph of $H_{R}$, and any two components in $R$ differ only by the choice of a matching on $S$. Each such matching has $\frac{n}{6}$ edges. Thus the number of components of $G$ in $R$ is at most

$$
\left(\begin{array}{c}
\left|E\left(H_{R}\right)\right| \\
n / 6
\end{array}\right) \leq\left(\begin{array}{c}
c d n^{4 / 3} \\
n / 6
\end{array}\right) \leq\left(\frac{\mathrm{e} c d n^{4 / 3}}{n / 6}\right)^{n / 6} \leq(6 \mathrm{e} c d)^{n / 6} n^{n / 18}
$$

Hence $|\mathcal{M}|<\left(2 d^{2}\right)^{n} \cdot(6 \mathrm{e} c d)^{n / 6} n^{n / 18}$, and by the lower bound on $|\mathcal{M}|$ from the start of the proof,

$$
\left(\frac{n}{9}\right)^{n / 6} \leq|\mathcal{M}|<\left(2 d^{2}\right)^{n} \cdot(6 \mathrm{e} c d)^{n / 6} n^{n / 18}
$$

The desired contradiction follows for all $n \geq\left(3456 \mathrm{e} c d^{13}\right)^{3 / 2}$.

\subsection{Graphs with bounded degree and bounded treewidth}

This section proves a logarithmic upper bound on the distance-number of graphs with bounded degree and bounded treewidth. Treewidth is an important parameter in Robertson and Seymour's theory of graph minors and in algorithmic complexity (see the surveys $[8,41])$. It can be defined as follows. A graph $G$ is a $k$-tree if either $G=K_{k+1}$, or $G$ has a vertex $v$ whose neighbourhood is a clique of order $k$ and $G-v$ is a $k$-tree. For 
example, every 1-tree is a tree and every tree is a 1-tree. Then the treewidth of a graph $G$ is the minimum integer $k$ for which $G$ is a subgraph of a $k$-tree. The pathwidth of $G$ is the minimum $k$ for which $G$ is a subgraph of an interval ${ }^{5}$ graph with no clique of order $k+2$. Note that an interval graph with no $(k+2)$-clique is a special case of a $k$-tree, and thus the treewidth of a graph is at most its pathwidth.

Lemma 7 shows that (1-)trees have bounded distance-number. However, this is not true for 2-trees since $K_{2, n}$ has treewidth (and pathwidth) at most 2 . By Theorem 3, there are $n$-vertex graphs of bounded degree with distance-number approaching $\Omega\left(n^{0.864138}\right)$. On the other hand, no polynomial lower bound holds for graphs of bounded degree and bounded treewidth, as shown in the following theorem.

Theorem 5. Let $G$ be a graph with $n$ vertices, maximum degree $\Delta$, and treewidth $k$. Then the distance-number of $G$ satisfies

$$
\operatorname{dn}(G) \in \mathcal{O}\left(\Delta^{4} k^{3} \log n\right)
$$

To prove Theorem 5 we use the following lemma, the proof of which is readily obtained by inspecting the proof of Lemma 8 in [14]. An $H$-partition of a graph $G$ is a partition of $V(G)$ into vertex sets $V_{1}, \ldots, V_{t}$ such that $H$ is the graph with vertex set $V(H):=$ $\{1, \ldots, t\}$ where $i j \in E(H)$ if and only if there exists $v \in V_{i}$ and $w \in V_{j}$ such that $v_{i} v_{j} \in E(G)$. The width of an $H$-partition is $\max \left\{\left|V_{i}\right|: 1 \leq i \leq t\right\}$.

Lemma 16 ([14]). Let $H$ be a graph admitting a drawing $D$ with s distinct edge-slopes and $\ell$ distinct edge-lengths. Let $G$ be a graph admitting an $H$-partition of width $w$. Then the distance-number of $G$ satisfies

$$
\operatorname{dn}(G) \leq \operatorname{s\ell w}(w-1)+\left\lfloor\frac{w}{2}\right\rfloor+\ell
$$

Sketch of Proof. The general approach is to start with $D$ and then replace each vertex of $H$ by a sufficiently scaled down and appropriate rotated copy of the drawing of $K_{w}$ on a regular $w$-gon. The only difficulty is choosing the rotation and the amount by which to scale the $w$-gons so that we obtain a (non-degenerate) drawing of $G$. Refer to [14] for the full proof.

Proof of Theorem 5. Let $w$ be the minimum width of a $T$-partition of $G$ in which $T$ is a tree. The best known upper bound is $w \leq \frac{5}{2}(k+1)\left(\frac{7}{2} \Delta(G)-1\right)$, which was obtained by Wood [51] using a minor improvement to a similar result by an anonymous referee of the paper by Ding and Oporowski [12]. For each vertex $x \in V(T)$, there are at most $w \Delta$ edges of $G$ incident to vertices mapped to $x$. Hence we can assume that $T$ is a forest with maximum degree $w \Delta$, as otherwise there is an edge of $T$ with no edge of $G$ mapped to it, in which case the edge of $T$ can be deleted. Similarly, $T$ has at most $n$ vertices. Scheffler [44] proved that $T$ has pathwidth at most $\log (2 n+1)$; see [8]. Dujmović et al.

\footnotetext{
${ }^{5} \mathrm{~A}$ graph $G$ is an interval graph if each vertex $v \in V(G)$ can be assigned an interval $I_{v} \subset \Re$ such that $I_{w} \cap I_{v} \neq \emptyset$ if and only if $v w \in E(V)$.
} 
[14] proved that every tree $T$ with pathwidth $p \geq 1$ has a drawing with $\max \{\Delta(T)-1,1\}$ slopes and $2 p-1$ edge-lengths. Thus $T$ has a drawing with at most $\Delta w-1$ slopes and at most $2 \log (2 n+1)-1$ edge-lengths. By Lemma 16,

$$
\operatorname{dn}(G) \leq(\Delta w-1)(2 \log (2 n+1)-1) w(w-1)+\left\lfloor\frac{w}{2}\right\rfloor+2 \log (2 n+1)-1,
$$

which is in $\mathcal{O}\left(\Delta w^{3} \log n\right) \subseteq \mathcal{O}\left(\Delta^{4} k^{3} \log n\right)$.

Corollary 1. Any n-vertex graph with bounded degree and bounded treewidth has distancenumber $\mathcal{O}(\log n)$.

Since a path has a drawing with one slope and one edge-length, Lemma 16 with $s=\ell=1$ implies that every graph $G$ with a $P$-partition of width $k$ for some path $P$ has distance-number $\operatorname{dn}(G) \leq k\left(k-\frac{1}{2}\right)+1$.

\section{Bandwidth}

This section finds an upper bound on the distance-number in terms of the bandwidth. Let $G$ be a graph. A vertex ordering of $G$ is a bijection $\sigma: V(G) \rightarrow\{1,2, \ldots,|V(G)|\}$. The width of $\sigma$ is defined to be $\max \{|\sigma(v)-\sigma(w)|: v w \in E(G)\}$. The bandwidth of $G$, denoted by bw $(G)$, is the minimum width of a vertex ordering of $G$. The cyclic width of $\sigma$ is defined to be $\max \{\min \{|\sigma(v)-\sigma(w)|, n-|\sigma(v)-\sigma(w)|\}: v w \in E(G)\}$. The cyclic bandwidth of $G$, denoted by $\operatorname{cbw}(G)$, is the minimum cyclic width of a vertex ordering of $G$; see $[11,20,28,30,53]$. Clearly $\operatorname{cbw}(G) \leq \operatorname{bw}(G)$ for every graph $G$.

Lemma 17. For every graph $G$,

$$
\operatorname{dn}(G) \leq \operatorname{cbw}(G) \leq \operatorname{bw}(G)
$$

Proof. Given a vertex ordering $\sigma$ of an $n$-vertex $G$, position the vertices of $G$ on a regular $n$-gon in the order $\sigma$. We obtain a drawing of $G$ in which the length of each edge $v w$ is determined by

$$
\min \{|\sigma(v)-\sigma(w)|, n-|\sigma(v)-\sigma(w)|\}
$$

Thus the number of edge-lengths equals

$$
|\{\min \{|\sigma(v)-\sigma(w)|, n-|\sigma(v)-\sigma(w)|\}: v w \in E(G)\}|,
$$

which is at most the cyclic width of $\sigma$. The result follows.

Corollary 2. The distance-number of every $n$-vertex degree- $\Delta$ planar graph $G$ satisfies

$$
\operatorname{dn}(G) \leq \frac{15 n}{\log _{\Delta} n}
$$

Proof. Böttcher et al. [9] proved that bw $(G) \leq \frac{15 n}{\log _{\Delta} n}$. The result follows from Lemma 17 . 


\section{Cartesian Products}

This section discusses the distance-number of cartesian products of graphs. For graphs $G$ and $H$, the cartesian product $G \square H$ is the graph with vertex set $V(G \square H):=V(G) \times V(H)$, where $(v, w)$ is adjacent to $(p, q)$ if and only if (1) $v=p$ and $w q$ is an edge of $H$, or $(2)$ $w=q$ and $v p$ is an edge of $G$.

Thus $G \square H$ is the grid-like graph with a copy of $G$ in each row and a copy of $H$ in each column. Type (1) edges form copies of $H$, and type (2) edges form copies of $G$. For example, $P_{n} \square P_{n}$ is the planar grid, and $C_{n} \square C_{n}$ is the toroidal grid.

The cartesian product is associative and thus multi-dimensional products are well defined. For example, the $d$-dimensional product $K_{2} \square K_{2} \square \ldots \square K_{2}$ is the $d$-dimensional hypercube $Q_{d}$. It is well known that $Q_{d}$ is a unit-distance graph. Horvat and Pisanski [24] proved that the cartesian product operation preserves unit-distance graphs. That is, if $G$ and $H$ are unit-distance graphs, then so is $G \square H$, as illustrated in Figure 7 . The following theorem generalises this result.

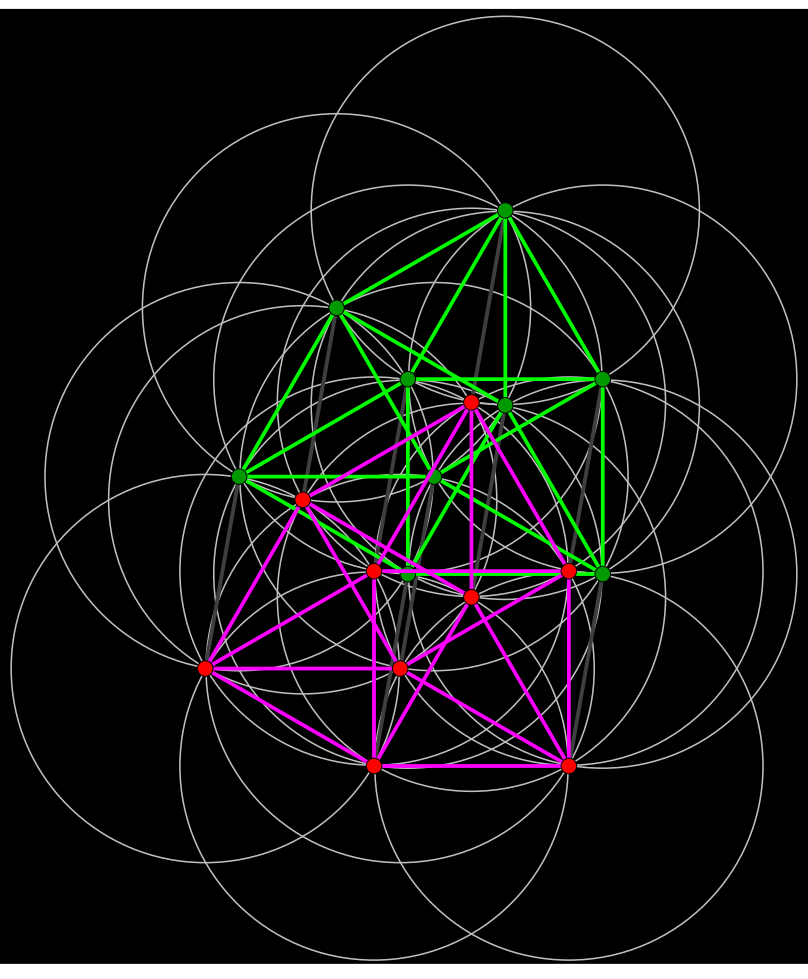

Figure 7: A unit-distance drawing of $K_{3} \square K_{3} \square K_{2}$

Theorem 6. For all graphs $G$ and $H$, the distance-numbers of $G \square H$ satisfy

$$
\begin{aligned}
\max \{\operatorname{ddn}(G), \operatorname{ddn}(H)\} & \leq \operatorname{ddn}(G \square H) \leq \operatorname{ddn}(G)+\operatorname{ddn}(H)-1, \text { and } \\
\max \{\operatorname{dn}(G), \operatorname{dn}(H)\} & \leq \operatorname{dn}(G \square H) \leq \operatorname{dn}(G)+\operatorname{dn}(H)-1 .
\end{aligned}
$$


Proof. The lower bounds follow since $G$ and $H$ are subgraphs of $G \square H$. We prove the upper bound for $\operatorname{dn}(G \square H)$. The proof for $\operatorname{ddn}(G \square H)$ is simpler.

Fix a drawing of $G$ with $\operatorname{dn}(G)$ edge-lengths. Let $(\mathrm{x}(v), \mathrm{y}(v))$ be the coordinates of each vertex $v$ of $G$ in this drawing. Fix a drawing of $H$ with $\operatorname{dn}(H)$ edge-lengths, scaled so that one edge-length in the drawing of $G$ coincides with one edge-length in the drawing of $H$. Let $\alpha$ be a real number in $[0,2 \pi)$. Let $\left(\mathrm{x}_{\alpha}(w), \mathrm{y}_{\alpha}(w)\right)$ be the coordinates of each vertex $w$ of $G$ in this drawing of $H$ rotated by $\alpha$ degrees about the origin.

Position vertex $(v, w)$ in $G \square H$ at $\left(\mathrm{x}(v)+\mathrm{x}_{\alpha}(w), \mathrm{y}(v)+\mathrm{y}_{\alpha}(w)\right)$. This mapping preserves edge-lengths. In particular, the length of a type-(1) edge $(v, u)(v, w)$ equals the length of the edge $u w$ in $H$, and the length of a type-(2) edge $(u, v)(w, v)$ equals the length of the edge $u w$ in $G$. Thus for each $\alpha$, the mapping of $G \square H$ has $\operatorname{dn}(G)+\operatorname{dn}(H)-1$ edge-lengths.

It remains to prove that for some $\alpha$ the mapping of $G \square H$ is a drawing. That is, no vertex intersects the closure of an incident edge. An angle $\alpha$ is bad for a particular vertex/edge pair of $G \square H$ if that vertex intersects the closure of that edge in the mapping with rotation $\alpha$.

Observe that the trajectory of a vertex $(v, w)$ of $G \square H$ (taken over all $\alpha$ ) is a circle centred at $(\mathbf{x}(v), \mathrm{y}(v))$ with radius $\operatorname{dist}_{H}(0, w)$.

Now for distinct points $p$ and $q$ and a line $\ell$, there are only two angles $\alpha$ such that the rotation of $p$ around $q$ by an angle of $\alpha$ contains $\ell$ (since the trajectory of $p$ is a circle that only intersects $\ell$ in two places), and there are only two angles $\alpha$ such that the rotation of $\ell$ around $q$ by an angle of $\alpha$ contains $p$.

It follows that there are finitely many bad values of $\alpha$ for a particular vertex/edge pair of $G \square H$. Hence there are finitely many bad values of $\alpha$ in total. Hence some value of $\alpha$ is not bad for every vertex/edge pair in $G \square H$. Hence $D_{\alpha}$ is a valid drawing of $G \square H$.

Note that Loh and Teh [31] proved a result analogous to Theorem 6 for dimension.

Let $G^{d}$ be the $d$-fold cartesian product of a graph $G$. The same construction used in Theorem 6 proves the following:

Theorem 7. For every graph $G$ and integer $d \geq 1$, the distance-numbers of $G^{d}$ satisfy

$$
\operatorname{ddn}\left(G^{d}\right)=\operatorname{ddn}(G) \quad \text { and } \quad \operatorname{dn}\left(G^{d}\right)=\operatorname{dn}(G) .
$$

\section{Open Problems}

We conclude by mentioning some of the many open problems related to distance-number.

- What is $\operatorname{dn}\left(K_{n}\right)$ ? Pach and Agarwal [37] write that "it can be conjectured" that $\operatorname{dn}\left(K_{n}\right)=\left\lfloor\frac{n}{2}\right\rfloor$. That is, every set of $n$ points in general position determine at least $\left\lfloor\frac{n}{2}\right\rfloor$ distinct distances. Note that Altman $[1,2]$ proved this conjecture for points in convex position.

- What is the relationship between distance-number and degenerate distance-number? In particular, is there a function $f$ such that $\operatorname{dn}(G) \leq f(\operatorname{ddn}(G))$ for every graph $G ?$ 
- Theorems 2, 3 and 4 establish a lower bound for the distance-number of bounded degree graphs. But no non-trivial upper bound is known. Do $n$-vertex graphs with bounded degree have distance-number in $o(n)$ ?

- Outerplanar graphs have distance-number in $\mathcal{O}\left(\Delta^{4} \log n\right)$ by Theorem 5 . Do outerplanar graphs (with bounded degree) have bounded (degenerate) distance-number?

- Non-trivial lower and upper bounds on the distance-numbers are not known for many other interesting graph families including: degree-3 graphs, degree-4 graphs, 2-degenerate graphs with bounded degree, graphs with bounded degree and bounded pathwidth.

- As described in Section 1.1, determining the maximum number of times the unitdistance can appear among $n$ points in the plane is a famous open problem. We are unaware if the following apparently simpler tasks have been attempted: Determine the maximum number of times the unit-distance can occur among $n$ points in the plane such that no three are collinear. Similarly, determine the maximum number of edges in an $n$-vertex graph $G$ with $\operatorname{dn}(G)=1$.

- Determining the maximum chromatic number of unit-distance graphs in $\Re^{d}$ is a well-known open problem. The best known upper bound of $(3+o(1))^{d}$ is due to Larman and Rogers [29]. Exponential lower bounds are known [17, 40]. Unitdistance graphs in the plane are 7 -colourable [19], and thus $\chi(G) \leq 7^{\mathrm{ddn}(G)}$. Can this bound be improved?

- Degenerate distance-number is not bounded by any function of dimension since $K_{n, n}$ has dimension 4 and unbounded degenerate distance-number. On the other hand, $\operatorname{dim}(G) \leq 2 \cdot \chi(G) \leq 2 \cdot 7^{\operatorname{ddn}(G)}$. Is $\operatorname{dim}(G)$ bounded by a polynomial function of $\operatorname{ddn}(G) ?$

- Every planar graph has a crossing-free drawing. A long standing open problem involving edge-lengths, due to Harborth et al. [21, 22, 26], asks whether every planar graph has a crossing-free drawing in which the length of every edge is an integer. Geelen et al. [18] recently answered this question in the affirmative for cubic planar graphs. Archdeacon [3] extended this question to nonplanar graphs and asked what is the minimum $d$ such that a given graph has a crossing-free drawing in $\Re^{d}$ with integer edge-lengths. Note that every $n$-vertex graph has such a drawing in $\Re^{n-1}$.

- The slope number of a graph $G$, denoted by $\operatorname{sn}(G)$, is the minimum number of edgeslopes over all drawings of $G$. Dujmović et al. [13] established results concerning the slope-number of planar graphs. Keszegh et al. [27] proved that degree-3 graphs have slope-number at most 5. On the other hand, Barát et al. [4] and Pach and Pálvölgyi [38] independently proved that there are 5-regular graphs with arbitrarily large slope number. Moreover, for $\Delta \geq 7$, Dujmović et al. [14] proved that there are $n$-vertex degree- $\Delta$ graphs whose slope number is at least $n^{1-\frac{\varepsilon}{\Delta+4}}$. The proofs of these 
results are similar to the proofs of Theorems 2, 3 and 4. Given that Theorem 5 also depends on slopes, it is tempting to wonder if there is a deeper connection between slope-number and distance-number. For example, is there a function $f$ such that $\operatorname{sn}(G) \leq f(\Delta(G), \operatorname{dn}(G))$ and/or $\operatorname{dn}(G) \leq f(\operatorname{sn}(G))$ for every graph $G$. Note that some dependence on $\Delta(G)$ is necessary since $\operatorname{sn}\left(K_{1, n}\right) \rightarrow \infty$ but $\operatorname{dn}\left(K_{1, n}\right)=1$.

\section{References}

[1] E. Altman. On a problem of P. Erdős. Amer. Math. Monthly, 70:148-157, 1963.

[2] E. Altman. Some theorems on convex polygons. Canad. Math. Bull., 15:329-340, 1972.

[3] Dan Archdeacon. Planar graphs with integer length straight edges, 1995. http://www. emba. uvm.edu/ archdeac/problems/integer.htm.

[4] JÁnos Barát, Jiří Matoušek, and David R. Wood. Bounded-degree graphs have arbitrarily large geometric thickness. Electron. J. Combin., 13(1):R3, 2006.

[5] Maria Belk. Realizability of graphs in three dimensions. Discrete Comput. Geom., 37:139-162, 2007.

[6] Maria Belk and Robert Connelly. Realizability of graphs. Discrete Comput. Geom., 37:125-137, 2007.

[7] Edward A. Bender And E. Rodney CAnfield. The asymptotic number of labeled graphs with given degree sequences. J. Combin. Theory Ser. A, 24:296-307, 1978.

[8] Hans L. Bodlaender. A partial $k$-arboretum of graphs with bounded treewidth. Theoret. Comput. Sci., 209(1-2):1-45, 1998.

[9] Julia Böttcher, Klaas P. Pruessmann, Anusch Taraz, and Andreas Würfl. Bandwidth, treewidth, separators, expansion, and universality. In Proc. Topological and Geometric Graph Theory (TGGT '08). 2008.

[10] Fred Buckley and Frank Harary. On the Euclidean dimension of a wheel. Graphs Combin., 4(1):23-30, 1988.

[11] Wai Hong Chan, Peter C. B. Lam, and Wai Chee Shiu. Cyclic bandwidth with an edge added. Discrete Appl. Math., 156(1):131-137, 2008.

[12] Guoli Ding and Bogdan Oporowski. Some results on tree decomposition of graphs. J. Graph Theory, 20(4):481-499, 1995.

[13] Vida Dujmović, David Eppstein, Matthew Suderman, and David R. Wood. Drawings of planar graphs with few slopes and segments. Comput. Geom., 38:194-212, 2007.

[14] Vida Dujmović, Matthew Suderman, and David R. Wood. Graph drawings with few slopes. Comput. Geom., 38:181-193, 2007. 
[15] Paul Erdős. On sets of distances of $n$ points. Amer. Math. Monthly, 53:248-250, 1946.

[16] Paul Erdős, Frank Harary, and William T. Tutte. On the dimension of a graph. Mathematika, 12:118-122, 1965.

[17] Peter Frankl and Richard M. Wilson. Intersection theorems with geometric consequences. Combinatorica, 1(4):357-368, 1981.

[18] Jim Geelen, Anjie Guo, And David McKinnon. Straight line embeddings of cubic planar graphs with integer edge lengths. J. Graph Theory, 58(3):270-274, 2008.

[19] Hugo Hadwiger and Hans Debrunner. Combinatorial geometry in the plane. Holt, New York, 1964.

[20] Frank Harary, Paul C. Kainen, and Adrian Riskin. Every graph of cyclic bandwidth 3 is toroidal. Bull. Inst. Combin. Appl., 27:81-84, 1999.

[21] Heiko Harborth And Arnfried Kemnitz. Integral representations of graphs. In Contemporary methods in graph theory, pp. 359-367. Bibliographisches Inst., Mannheim, 1990.

[22] Heiko Harborth, Arnfried Kemnitz, Meinhard Möller, and Andreas Süssenbach. Ganzzahlige planare Darstellungen der platonischen Körper. Elem. Math., 42(5):118-122, 1987.

[23] Robert Hochberg and Paul O'Donnell. Some 4-chromatic unit-distance graphs without small cycles. Geombinatorics, 5(4):137-141, 1996.

[24] Boris Horvat and Tomaž Pisanski. Products of unit distance graphs. In 6th Slovenian International Conference on Graph Theory. Bled, Slovenia, 2007.

[25] Nets Hawk Katz And GÁbor Tardos. A new entropy inequality for the Erdős distance problem. In Towards a theory of geometric graphs, vol. 342 of Contemp. Math., pp. 119-126. Amer. Math. Soc., 2004.

[26] Arnfried Kemnitz and Heiko Harborth. Plane integral drawings of planar graphs. Discrete Math., 236(1-3):191-195, 2001.

[27] Balázs Keszegh, Dömötör PÁlvölgyi, JÁnos Pach, And GÉza Tóth. Drawing cubic graphs with at most five slopes. Comput. Geom., 40(2):138-147, 2008.

[28] Peter C. B. Lam, Wai Chee Shiu, and Wai Hong Chan. Characterization of graphs with equal bandwidth and cyclic bandwidth. Discrete Math., 242(1-3):283$289,2002$.

[29] David G. Larman and C. Ambrose Rogers. The realization of distances within sets in Euclidean space. Mathematika, 19:1-24, 1972.

[30] Yi Xun Lin. The cyclic bandwidth problem. Systems Sci. Math. Sci., 7(3):282-288, 1994.

[31] Hool-tong Loh and Hoon Heng TeH. On the dimension of product graphs. Nanta Math., 1:68-71, 1966/1967. 
[32] Hiroshi Maehara. Note on induced subgraphs of the unit distance graph $E^{n}$. Discrete Comput. Geom., 4(1):15-18, 1989.

[33] Hiroshi Maehara And Vojtěch RöDl. On the dimension to represent a graph by a unit distance graph. Graphs Combin., 6(4):365-367, 1990.

[34] Brendan D. McKay. Asymptotics for symmetric 0-1 matrices with prescribed row sums. Ars Combin., 19(A):15-25, 1985.

[35] Paul O'Donnell. Arbitrary girth, 4-chromatic unit distance graphs in the plane. I. Graph description. Geombinatorics, 9(3):145-152, 2000.

[36] Paul O'Donnell. Arbitrary girth, 4-chromatic unit distance graphs in the plane. II. Graph embedding. Geombinatorics, 9(4):180-193, 2000.

[37] János Pach and Pankaj K. Agarwal. Combinatorial geometry. John Wiley \& Sons Inc., New York, 1995.

[38] JÁnos PACH AND DÖмÖтÖR PÁLvÖLGYI. Bounded-degree graphs can have arbitrarily large slope numbers. Electron. J. Combin., 13(1):N1, 2006.

[39] Dan Pritikin. All unit-distance graphs of order 6197 are 6-colorable. J. Combin. Theory Ser. B, 73(2):159-163, 1998.

[40] A. M. RaĬGorodskiĬ. On the chromatic number of a space. Uspekhi Mat. Nauk, 55(2):147-148, 2000.

[41] Bruce A. Reed. Algorithmic aspects of tree width. In Bruce A. Reed And Cláudia L. Sales, eds., Recent Advances in Algorithms and Combinatorics, pp. 85-107. Springer, 2003.

[42] Michael Reid. On the connectivity of unit distance graphs. Graphs Combin., 12(3):295-303, 1996.

[43] Lajos Rónyai, László Babai, and Murali K. Ganapathy. On the number of zero-patterns of a sequence of polynomials. J. Amer. Math. Soc., 14(3):717-735, 2001.

[44] Petra Scheffler. Die Baumweite von Graphen als ein Maß für die Kompliziertheit algorithmischer Probleme. Ph.D. thesis, Akademie der Wissenschaften der DDR, Berlin, Germany, 1989.

[45] Saharon Shelah and Alexander Soifer. Axiom of choice and chromatic number of the plane. J. Combin. Theory Ser. A, 103(2):387-391, 2003.

[46] József Solymosi, Gábor Tardos, and Csaba D. Tóth. The $k$ most frequent distances in the plane. Discrete Comput. Geom., 28(4):639-648, 2002.

[47] József Solymosi and Csaba D. Tóth. Distinct distances in the plane. Discrete Comput. Geom., 25(4):629-634, 2001.

[48] Joel H. Spencer, Endre Szemerédi, and William T. Trotter, Jr. Unit distances in the Euclidean plane. In BÉla BollobAs, ed., Graph theory and combinatorics, pp. 293-303. Academic Press, London, 1984. 
[49] LÁszLó A. SzÉKelY. Crossing numbers and hard Erdős problems in discrete geometry. Combin. Probab. Comput., 6(3):353-358, 1997.

[50] Gábor Tardos. On distinct sums and distinct distances. Adv. Math., 180(1):275289, 2003.

[51] David R. Wood. On tree-partition-width, 2007. arXiv.org/math/0602507.

[52] Nicholas Wormald. Some problems in the enumeration of labelled graphs. Ph.D. thesis, University of Newcastle, United Kingdom, 1978.

[53] Sanming Zhou. Bounding the bandwidths for graphs. Theoret. Comput. Sci., 249(2):357-368, 2000. 